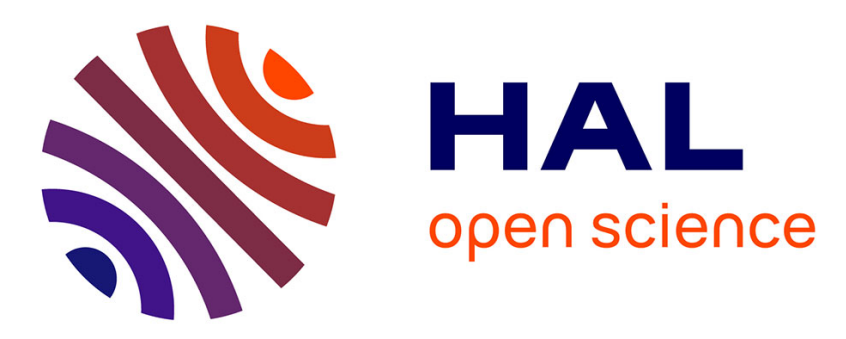

\title{
Slip events and dilatancy in a sheared fine noncohesive powder
}

\author{
M Lubert, Alain de Ryck
}

\section{To cite this version:}

M Lubert, Alain de Ryck. Slip events and dilatancy in a sheared fine noncohesive powder. Physical Review E , 2001, 63 (2, 1), pp.art. no.-021502. 10.1103/PhysRevE.63.021502 . hal-01652389

\section{HAL Id: hal-01652389 \\ https://hal.science/hal-01652389}

Submitted on 8 Nov 2019

HAL is a multi-disciplinary open access archive for the deposit and dissemination of scientific research documents, whether they are published or not. The documents may come from teaching and research institutions in France or abroad, or from public or private research centers.
L'archive ouverte pluridisciplinaire HAL, est destinée au dépôt et à la diffusion de documents scientifiques de niveau recherche, publiés ou non, émanant des établissements d'enseignement et de recherche français ou étrangers, des laboratoires publics ou privés. 


\title{
Slip events and dilatancy in a sheared fine noncohesive powder
}

\author{
Mylène Lubert and Alain de Ryck \\ École des Mines d'Albi, Campus Jarlard, 81013 Albi Cedex 09, France
}

\begin{abstract}
We present experimental results of the transition from steady-state sliding to oscillatory motion for a fine noncohesive powder, sheared in an annular cell. The onset of instability is compared to the Dieterich-Ruina model for solid friction. We present data showing that at low velocity and close to the transition, the major sliding jumps are preceded by a short or long period of unstable plastic yielding of the granular matter. This ambivalent behavior suggests that the jumps are initiated when the sliding overcomes a critical velocity. During the stick-slip motion, the dilatancy of the powder bed has been also observed: the slippage is associated with a compaction whose value increases with the jump in the friction coefficient.
\end{abstract}

PACS number(s): 45.70.Ht, 62.40.+i, 91.30.Px

\section{INTRODUCTION}

The shear behavior of a dry granular medium is important in silo design procedures $[1,2]$, for the understanding of earthquake dynamics [3-6] since the faults are generally filled by debris, and for fundamental aspects of granular mechanics.

At high shear rates, the stresses are due to the collisions between grains (Bagnold regime) [7-9]. Here we focus on the dense regime where the friction between grains dominates. In this case, the strain localizes in shear bands of a thickness of about ten particles and the shear stress follows the Amontons-Coulomb law $\tau=\mu \sigma$, where $\tau$ and $\sigma$ are, respectively, the tangential and normal stresses. $\mu$, the coefficient of friction, is a constant of order unity, which depends on the material.

As in friction between two solid bodies, shear is often not steady state but proceeds by stick and slip movements [3-6,10-12]. The transition to oscillatory motion has been observed in rock-friction experiments $[5,6]$ (normal stresses of order the $\mathrm{MPa}$ ) and at low pressures $(\mathrm{kPa})$ in paper-onpaper sliding experiments [10]. The instability occurs above a threshold in normal loading and disappears when using stiff enough shear testers.

The phenomenological description by Dieterich and Ruina $[3,4]$ of a coefficient of friction that decreases with the sliding velocity and increases with the time of contact between the two surfaces well describes this transition. The transition to stick-slip oscillations can also be described by replacing the time strengthening of the Dieterich-Ruina model by a dilatancy weakening [13-15].

Such stick-slip motion has been also observed for a granular medium sheared at the surface of a rough plate [12]. This latter system allowed direct observation of precursor events inducing a small creep before the slip.

Here, we report some experiments where the transition from steady-state sliding to stick-slip motion is observed when a fine noncohesive powder is sheared in the bulk. In Sec. II, the material and the apparatus used are presented. Section III presents the experimental results and Sec. IV discusses them. Three points are successively commented: (1) the onset of shear instability and its fit with the DieterichRuina model for solid friction, (2) the observation of a new transition to regular stick-slip oscillations, with a coexistence of yield and slip states and (3) a simple sliding velocity criterion is derived to explain our experimental data.

\section{EXPERIMENTAL SETUP}

A. The powder

We use a silica gel ground and sieved between 40 and 63 $\mu \mathrm{m}$ (Fig. 1). Due to its microporosity (mean diameter of the pores $60 \AA$ ), this powder is only slightly sensitive to changes in ambient atmospheric relative humidity and gives very reproducible results.

\section{B. The apparatus}

The experimental setup is an annular shear tester (from Schulze Schüttgutmeßtechnik, D-38302 Wolfenbüttel), sketched in Fig. 2. The powder is put inside a ring of thickness $4 \mathrm{~cm}$, which rotates at the angular velocity $\omega$ (ranging from $10^{-4}$ to $5 \times 10^{-3} \mathrm{rad} / \mathrm{s}$ ). The cover plate is prevented from following the rotation by using two force transducers (strain gauge) as stops. Their distance from the axis of rotation is $L=12.5 \mathrm{~cm}$ and their apparent stiffness is $k_{0}$ $=1.0 \pm 0.110^{5} \mathrm{~N} / \mathrm{m}$. The shear torque measured is

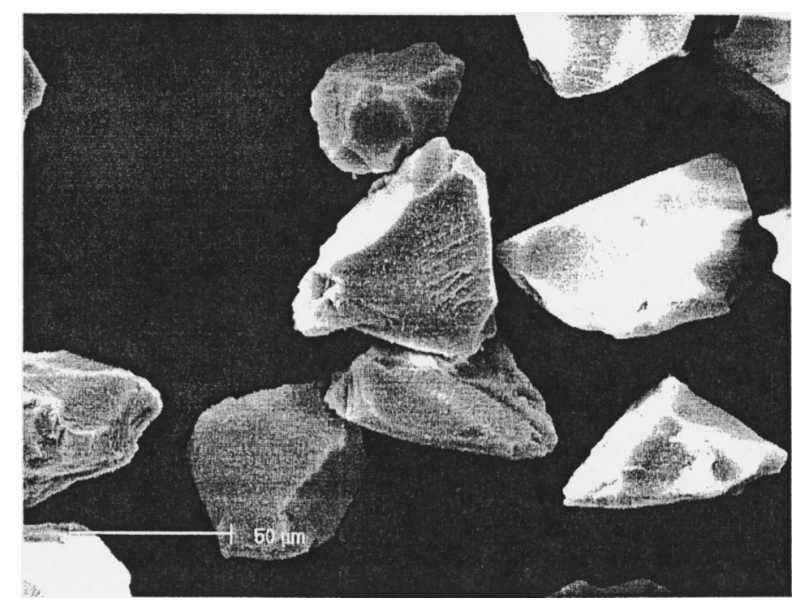

FIG. 1. Scanning electron microscopy of the silicagel grains. The bar represents $50 \mu \mathrm{m}$. 


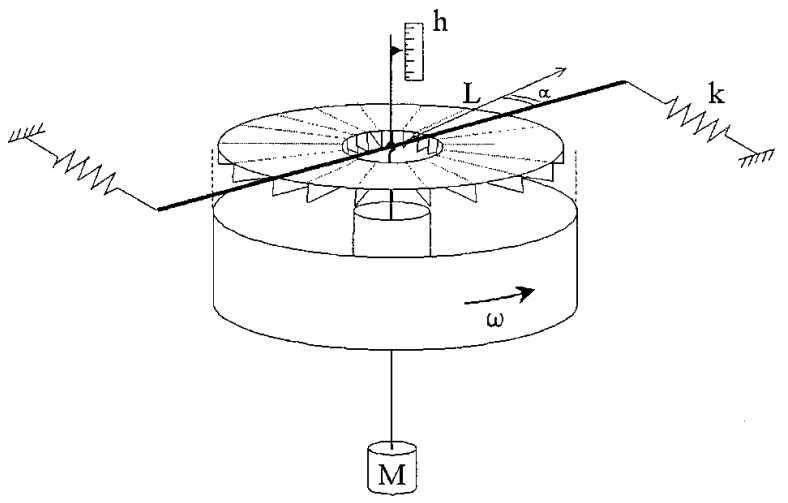

FIG. 2. Sketch of the experimental setup. Two annular shear cells have been used: a big cell of inner radius $5 \mathrm{~cm}$, outer radius 10 $\mathrm{cm}$, and depth $4 \mathrm{~cm}$; a small cell of inner radius $3 \mathrm{~cm}$, outer radius $6 \mathrm{~cm}$, and depth $2.5 \mathrm{~cm}$.

$\Gamma=2 k_{0} L^{2} \alpha_{0}$, where $\alpha_{0}$ is the angular displacement of the cover plate. It also writes $\Gamma=2 k L^{2} \alpha$, where $\alpha=\alpha_{0}+c \Gamma$ adds the elastic angular displacement of the powder sample and where $k=k_{0} /\left(1+2 k_{0} L^{2} c\right)$ is the apparent stiffness of the whole system. In order to shear inside the bulk, the cover plate has 20 teeth of $5-\mathrm{mm}$ depth. These small steel teeth drive into the granular material and push it. The state of stresses and compaction is not constant along the shear plane (located at the end of the teeth), but depends on the distance from the teeth. We have checked that the number of teeth employed (from 20 to only 2) does not change the friction observed in permanent shearing (steady-state or oscillatory). Nevertheless, we can notice that in the case of only 2 teeth symmetrically disposed, it takes more time to reach this regime.

On the cover plate is hooked a pendulum of length $l$ $=70 \mathrm{~cm}$, which takes weights from $M_{0}=100 \mathrm{~g}$ to $25 \mathrm{~kg}$ (Fig. 2). We have checked a posteriori that the oscillating period $2 \pi \sqrt{l / g}=1.7 \mathrm{~s}$ of this pendulum does not induce any resonance effects with the shear oscillations studied. In order to reach small normal stresses, the cover plate and the pendulum are counterweighted (not represented on the sketch of Fig. 2).

This annular geometry has some advantages: we are not limited in strain and the vertical displacement of the cover plate, measured by an inductive transducer, is more homogeneous. However, some powder is extruded from the gap of 1 $\mathrm{mm}$ between the cover and the side walls of the cell during the experiments. This leads to a decrease of the height of the sample of about $1 \mathrm{~mm}$ per revolution (half a micron in $14 \mathrm{~s}$, the typical time of our recordings reported in the manuscript). Another disadvantage is that the shear velocity is not constant over the whole shear plane. It doubles from the inner to the outer radius since the cell used has inner and outer radii of 5 and $10 \mathrm{~cm}$ (we also use a small cell with inner radius $R_{i}=3 \mathrm{~cm}$, outer radius $R_{0}=6 \mathrm{~cm}$, and thickness $2.5 \mathrm{~cm})$.

\section{Experimental procedure}

For all the experiments, the silicagel powder was poured inside the annulus and the surface leveled by a knife. The sample is then sheared with the desired velocity and normal loading until it reaches a permanent steady or oscillatory state. For a series of experiments, the motor speed and normal weights have been changed without removing the sample. We check the reproducibility of our experiments by using several samples.

\section{The measurements}

Data are recorded by sampling the shear torque and the dilatancy signals at 500 points per second, with 4 digit precision. For the dilatancy, the signal is filtered by a mobile averaging on 5 points $(10 \mathrm{~ms})$.

The torque and the normal load on the shear plane are given by

$$
\Gamma_{\text {friction }}=\int_{0}^{2 \pi} d \theta \int_{R_{i}}^{R_{o}} d r \tau r^{2}
$$

and

$$
M g=\frac{1}{g} \int_{0}^{2 \pi} d \theta \int_{R_{i}}^{R_{o}} d r \sigma r,
$$

where $(r, \theta)$ are the polar coordinates. We have $M=M_{0}$ $+m$, where $m$ is the mass of the powder between the teeth. For the silicagel, with a measured dense bulk density of 550 $\mathrm{kg} / \mathrm{m}^{3}$, it leads to $m=65 \mathrm{~g}$.

Assuming the Amontons-Coulomb law on the shear plane, $\tau=\mu \sigma$, and that the stresses depend only on the angular position $\theta$, due to the teeth and not on the radial position, we obtain

$$
\Gamma_{\text {friction }}=\frac{2}{3} \frac{R_{o}^{3}-R_{i}^{3}}{R_{o}^{2}-R_{i}^{2}} \mu M g .
$$

The equation of the dynamics for the cover plate is

$$
J \ddot{\alpha}_{o}=-\Gamma+\Gamma_{\text {friction }},
$$

where $J$ is the inertial moment of the cover plate and $\Gamma$ the shear torque measured by the sensors. In Eq. (3), the inertial term remains negligible while the typical time for the variations $\sqrt{\alpha / \ddot{\alpha}}$ remains greater than the inertial time $t_{\text {in }}$ $=\sqrt{J / 2 k_{0} L^{2}} \sim \sqrt{M_{\text {cover }} / k_{0}}$, of the order of $5 \mathrm{~ms}$, which is the case for our experiments. $\mu$ is then related to the angular displacement $\alpha$ by

$$
\mu=\frac{k}{M g} 3 L^{2} \frac{R_{o}^{2}-R_{i}^{2}}{R_{o}^{3}-R_{i}^{3}} \alpha .
$$

\section{EXPERIMENTAL RESULTS}

\section{A. Shear oscillations}

Figure 3 presents the time recording of the friction coefficient of the silicagel in the permanent shearing regime for normal loads ranging from 2 to $24 \mathrm{~kg}$. For clarity, the curves have been shifted with respect to each other since the maximum of $\mu$ is always around $0.65 \pm 0.02$. The upper scale 


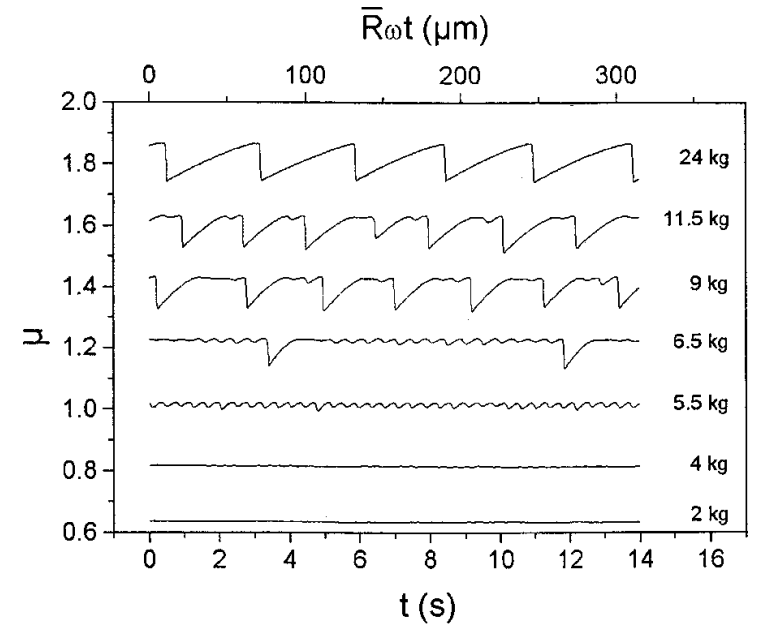

FIG. 3. Frictional coefficient $\mu$ versus time for different normal loads $M$ (big cell, $\omega_{0}=3 \times 10^{-4} \mathrm{rad} / \mathrm{s}$ ). For the sake of clarity, the curves are shifted with respect to each other by a jump of 0.2 .

gives the displacement of the lower part of the cell calculated at the mean radius $\left(\bar{R}=7.5 \mathrm{~cm}\right.$ and $\left.\omega=3 \cdot 10^{-4} \mathrm{rad} / \mathrm{s}\right)$.

We observe four types of behavior when increasing the normal load: (i) For $M \leqslant 5 \mathrm{~kg}$ the shear force is steady state. (ii) At $5.5 \mathrm{~kg}$, some oscillations of small amplitude and finite period $\left(T_{c}=0.39 \mathrm{~s}\right.$, pulsation $\left.\Omega_{c}=2 \pi / T_{c}=16.1 \mathrm{rad} / \mathrm{s}\right)$ appear. During one oscillation, the relative displacement on the shear plane is $8.8 \mu \mathrm{m}$. (iii) For large loading $(M \geqslant 20 \mathrm{~kg}$, only $M=24 \mathrm{~kg}$ is represented in Fig. 3), the movement proceeds by regular stick and slip phases as in solid friction $[3,10]$. (iv) In between (curves for $M=6.5,9$, and $11.5 \mathrm{~kg}$ in Fig. 3), we observe a new behavior, where the slip phases are followed by a stick and then a yield phase, which is not stable and presents growing oscillations. For the $6.5 \mathrm{~kg}$ experiment, the sliding distance between the two slip events is about $200 \mu \mathrm{m}, 4$ times the particle diameter.

\section{B. Dilatancy}

During these shear experiments, the height of the sample has been recorded and is displayed on Fig. 4. In this figure, we find again the same succession of regimes as observed for the shear force in Fig. 3. (i) Below the instability threshold $(M \leqslant 5 \mathrm{~kg})$, there is no variation in the height of the sample except that due to extrusion of particles out of the cell. (ii) At $5.5 \mathrm{~kg}$, small oscillations of amplitude $0.2 \mu \mathrm{m}$ are observed. The period is the same as that for the force. It is also noticed in this curve that a knock given on the apparatus induces a small disturbance (at $3 \mathrm{~s}$ ) which quickly disappears and does not perturb the force signal. (iii) For $M=24 \mathrm{~kg}$, we observe that the big slippages are associated with a packing down of the powder of the order a fraction of the particle diameter. The over oscillations observed after the packing down are due to the weak damping of the measuring device. During the stick phase, the medium is dilating and rupture occurs while it is still expanding. (iv) When decreasing the mass from 24 to $6.5 \mathrm{~kg}$, we notice a decrease in the height jump during packing down and the appearance of an inflection in

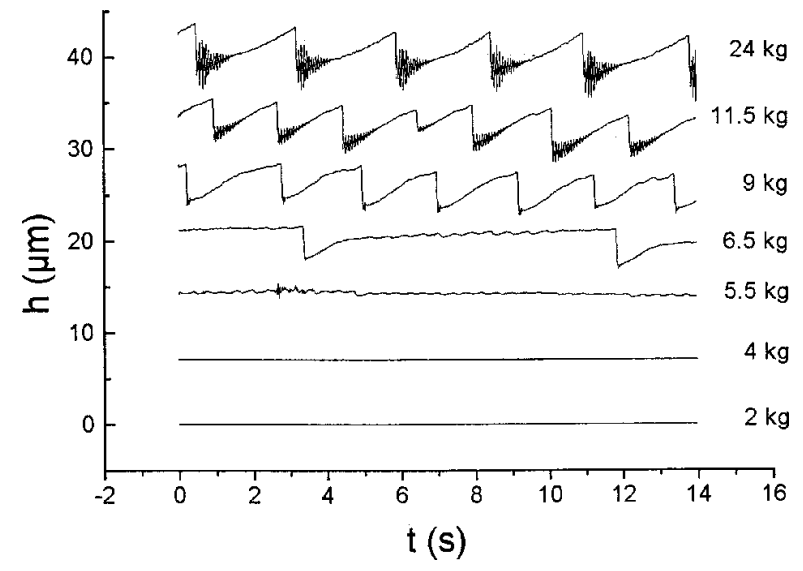

FIG. 4. Vertical displacement $h$ of the cover plate as a function of time corresponding to the experiments of (a). For the high masses, over oscillations of period $0.034 \mathrm{~s}$ appear due to the weak damping of the measuring device.

the dilatancy phase. At $6.5 \mathrm{~kg}$, the dilatancy has time to reach a plateau before the slip event occurs.

\section{DISCUSSION}

Though not all the experimental observations fit a solid friction behavior, we propose as a first approach to describe the instability onset with the aid of the Dieterich-Ruina model.

\section{A. The threshold}

The onset of oscillations at $5.5 \pm 0.5 \mathrm{~kg}$ is found to be independent on velocity as it can be observed in Fig. 5, where the amplitude of the oscillations of the coefficient of friction is plotted versus the normal load for three different velocities. On the other hand, the higher the driving velocity, the less is the amplitude. This amplitude decrease has been also observed in solid friction [10]. In Fig. 6, we compare the oscillating amplitude obtained for the small cell and the big cell with the same angular velocity. The threshold is higher for the small geometry, at around $16 \mathrm{~kg}$. This instability threshold is therefore not given by a critical normal stress

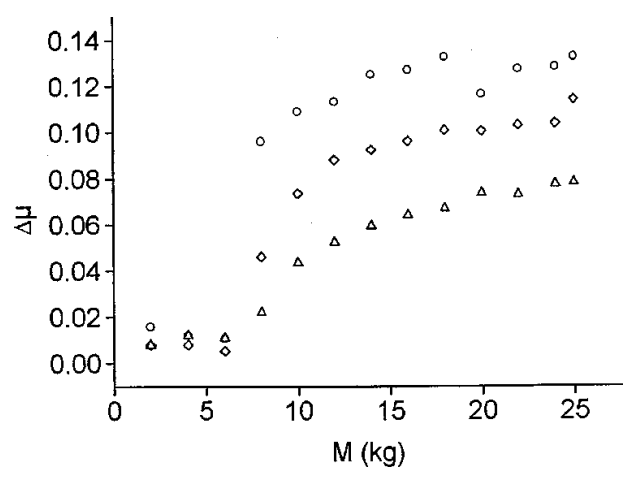

FIG. 5. Amplitude of the oscillations of $\mu$ as a function of the normal load for different velocities: circles: $\omega_{0}=3.7 \times 10^{-4} \mathrm{rad} / \mathrm{s}$; diamonds: $\omega_{0}=7.8 \times 10^{-4} \mathrm{rad} / \mathrm{s}$, and triangles: $3.4 \times 10^{-3} \mathrm{rad} / \mathrm{s}$. 


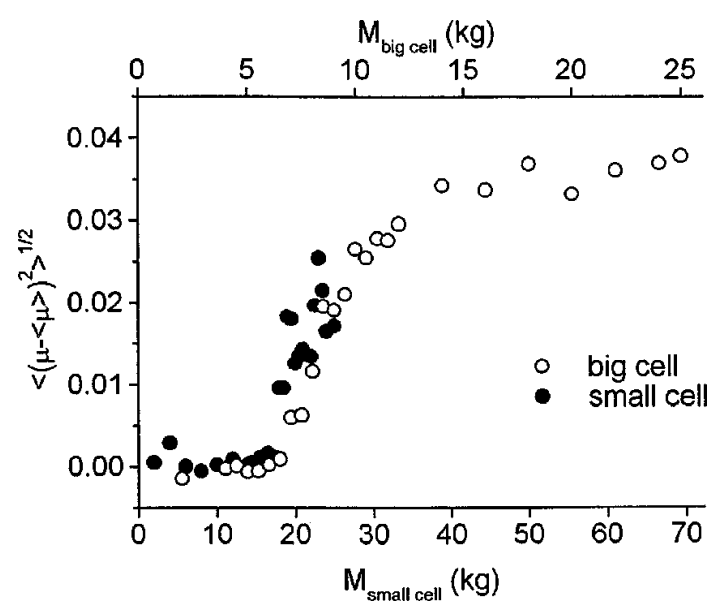

FIG. 6. Standard deviation of $\mu$ as a function of the normal load for two cells (black dots: small cell; open dots: big cell). The two $x$ axes are homothetic, by a factor corresponding to the $(5 / 3)^{2}$ geometrical coefficient of Eq. (7). The two sets of data then collapse to the same curve.

since, for the small cell, the instability develops for $\sigma$ greater than $19 \mathrm{kPa}$, and for the big cell, for $\sigma$ greater than $2.3 \mathrm{kPa}$.

This instability onset observed with this granular medium may be compared to that given by the Dieterich-Ruina model in solid friction experiments (for a mass $M$ pulled by a spring of stiffness $k$ at constant velocity). This model assumes that $[3,4]$ :

$$
\mu=\mu_{0}+a \ln \frac{V}{V_{0}}+b \ln \frac{V_{0} \theta}{D_{c}},
$$

with

$$
\frac{d \theta}{d t}=1-\frac{V \theta}{D_{c}}
$$

where $V$ is the sliding velocity. In our experimental setup, the velocity is not constant over the shear plane but the velocity dependence of the friction is only logarithmic. We approximate this sliding velocity by a constant: $V=\bar{R}\left(\omega_{0}\right.$ $-\dot{\alpha}$ ), where $\bar{R}$ is an average radius. $\mu_{0}$ is the steady-state friction coefficient at a reference velocity $V_{0}$ and $a$ and $b$ are two numerical constants that depend on the material. $D_{c}$ is the critical slip distance; it scales with the sliding distance necessary to renew the contact population. $\theta$ can be assimilated to a time of contact (at steady state, $\theta=D_{c} / V$ ). The first logarithmic term represents the instantaneous change in the friction coefficient when changing the sliding velocity, whereas the second term is representative of the time evolution.

Together with Eq. (4), we obtain a dynamic system, which linear stability analysis shows to become unstable for $[3,10]$ :

$$
\frac{k}{M g} \leqslant \beta \frac{b-a}{D_{c}}
$$

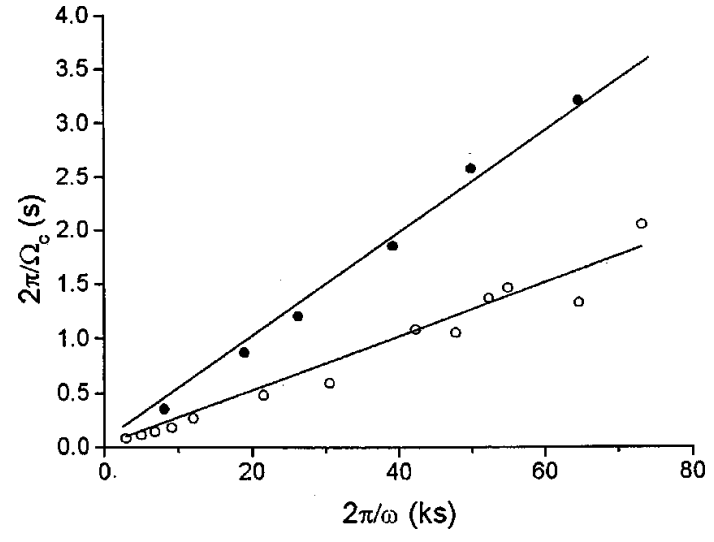

FIG. 7. Period of the oscillations at threshold as a function of the period of rotation of the cell (black dots: small cell and normal load $15 \mathrm{~kg}$; open dots: big cell and normal load $5 \mathrm{~kg}$ ).

where $\beta$ is a coefficient that depends on the geometry. For the spring-block system, $\beta=1$, and in the cylindrical geometry, we have

$$
\beta=\frac{1}{3} \frac{\bar{R}}{L^{2}} \frac{R_{0}^{3}-R_{i}^{3}}{R_{0}^{2}-R_{i}^{2}} .
$$

This geometrical effect has been checked. In Fig. 6, we have adjusted the two $x$-axis scales in order to fit the $(5 / 3)^{2}$ ratio between the coefficients $\beta$ for the two cells (respectively, 0.187 and 0.0672 for the big and small one). The amplitudes then fall on the same curve. This gives some confidence in the use of the Dieterich-Ruina model.

\section{Determination of the parameters of the model}

Introducing the critical mass $M^{*}=5.5 \mathrm{~kg}$ at threshold in Eq. (6) and the stiffness of the whole system (apparatus and powder) measured experimentally: $k=6 \times 10^{4} \mathrm{~N} / \mathrm{m}$, we obtain a value of $170 \pm 20 \mu \mathrm{m}$ for $D_{c} /(b-a)$.

The pulsation $\Omega_{c}$ of the oscillations at threshold is also given by the linear stability analysis $[3,10]$ and leads to

$$
\Omega_{c}=\frac{\bar{R}}{D_{c}} \omega \sqrt{\frac{b-a}{a}} .
$$

This proportionality with the rotational velocity of the cell has been checked experimentally for the two cells as shown in Fig. 7 where the periods of the oscillations at threshold is plotted versus the period of rotation of the shear cell. Using Eq. (6) and Eq. (8), a second relation can be evaluated:

$$
a D_{c}=\frac{\bar{R}^{2}}{\beta} \frac{k}{M^{*} g}\left(\frac{\omega}{\Omega_{c}}\right)^{2} .
$$

Using the values obtained for the same experiment leads to $a D_{c}=0.012 \pm 0.005 \mu \mathrm{m}$. But, in order to evaluate separately the three parameters $a, b$, and $D_{c}$ of the model, we need another experiment.

The coefficient $b$ has been directly determined by holding experiments [5], which consist of restarting the rotation after 


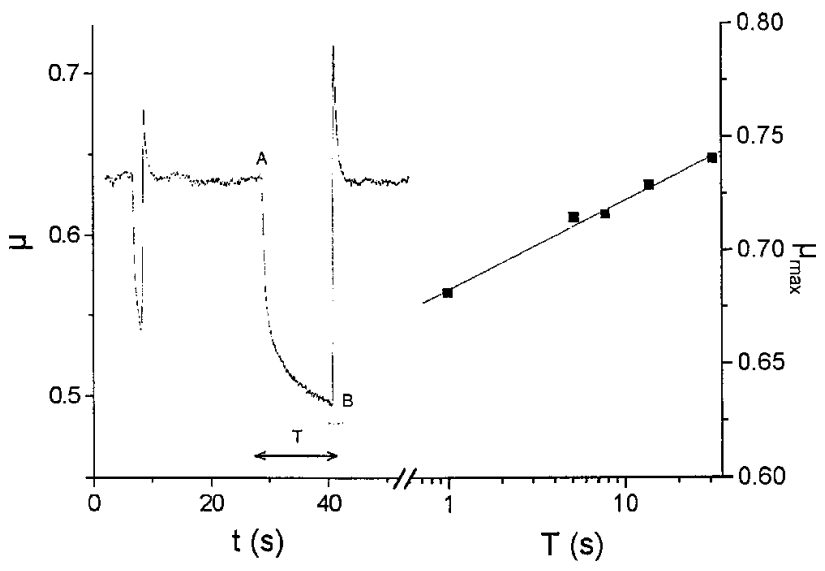

FIG. 8. Left part: temporal plot of the friction coefficient for $M=400 \mathrm{~g}$. In A, the motor is stopped. In B the shear is resumed after the holding time $T$. When resuming the shear, the friction coefficient goes through a maximum $\mu_{\max }$. Right part: semilog plot of $\mu_{\max }$ versus $T$.

a period of rest $T$ and measuring the maximum of friction $\mu_{\max }$ reached. The left-hand side of Fig. 8 presents an experimental curve with two holds of 1 and $13 \mathrm{~s}$ using a normal load of $400 \mathrm{~g}$. The right-hand side is a semilogarithmic plot of $\mu_{\max }$ versus $T$. The slope $d \mu_{\max } / d \ln T$ gives $b$ since without sliding ( $V=0), \theta$ in Eq. (5) identifies to the usual time. We find $b=0.017 \pm 0.005$.

From this last determination, we obtain a critical slip length $D_{c}$ of $1.4 \pm 0.2 \mu \mathrm{m}$. This value is comparable to those observed in solid friction $[3-5,10]$ and much smaller than the diameter of the grains. This suggests that the grains do not play the role of the roughness in solid friction and that the onset of instability is due to contacts between particles.

From these measurements, we also obtain $b-a=0.008$ \pm 0.005 . This difference may be measured directly by looking at the variation of $\mu$ with the velocity at steady-state sliding (for weak normal loading) [3]. The experiments actually give a very low dependence of $\mu$ with $\omega$, as shown in Fig. 9. In the same figure, a linear regression in the semilog

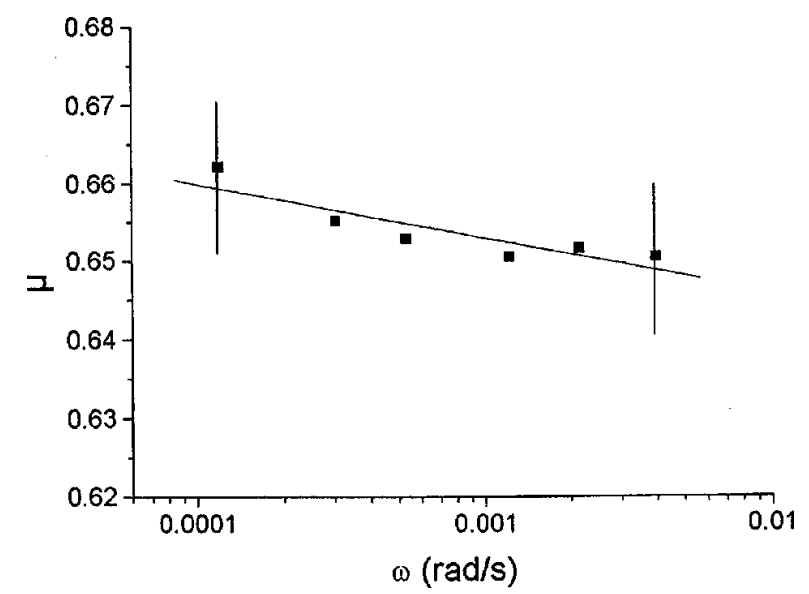

FIG. 9. Semilog plot of the steady-state friction coefficient versus the angular velocity $\omega$. Here $M=1 \mathrm{~kg}$.

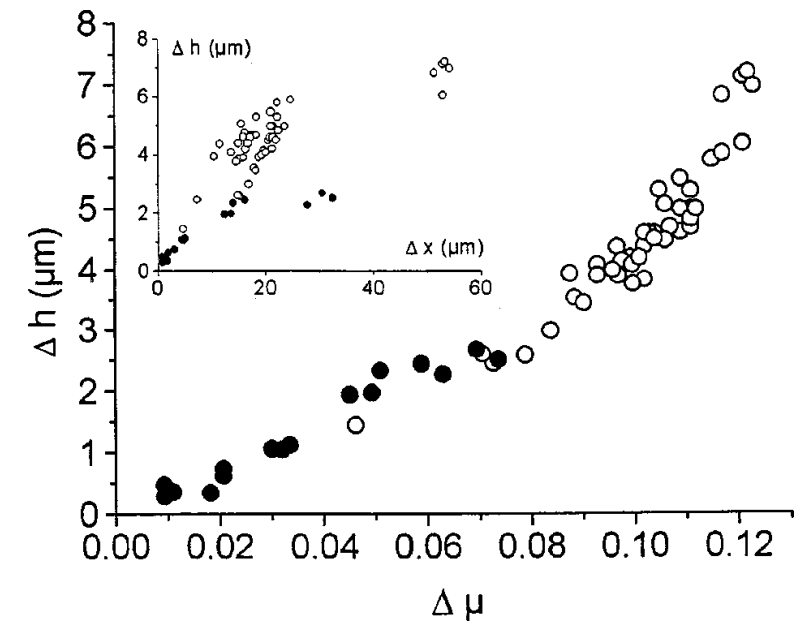

FIG. 10. Vertical jump compaction $\Delta h$ as a function of the horizontal slippage $\Delta \mu$ for two shear velocities (open dots: $\omega_{0}$ $=3 \times 10^{-4} \mathrm{rad} / \mathrm{s}$, experiments of Fig. 3 ; black dots: $\omega_{0}$ $=3.5 \times 10^{-3} \mathrm{rad} / \mathrm{s}$, experiments of Fig. 13). Insert: the same $\Delta h$ data are plotted versus the horizontal displacement $\Delta x$.

scale gives a value $b-a=-d \mu_{s s} / d \ln \omega=0.003 \pm 0.001$. This weak variation is of the order of the precision of the setup but is not in contradiction with the other determinations.

We conclude the observation of the instability threshold for a granular medium may be described by the DieterichRuina model for solid friction. The critical length $D_{c}$ deduced from the model is of the order of a micron, thus suggesting that the instability is triggered by grain-grain friction and not by grain rearrangements.

\section{B. The transition}

After the threshold, we observe a coexistence of two different kinds of behavior: small oscillations of the shear force, similar to those at the threshold, and big rearrangements, as for $M=24 \mathrm{~kg}$ (Fig. 3). When the loading is increased from the onset of instability, the duration of the yielding between two rearrangements becomes smaller and finally disappears. A succession of stick and slip phases then subsists, where the slip is preceded by a small creep as in Ref. [10].

To explain this mixed behavior during the transition, we first examine the role of dilatancy.

\section{Packing down during the slip events}

These big slippages produce an acoustic noise and are associated with a packing down of the powder by an order of a fraction of the particle diameter (Fig. 4). This packing down $\Delta h$ increases with the jump of friction coefficient $\Delta \mu$ as shown in Fig. 10. In this figure, the white points corresponds to the experiments of Fig. 3 and the black points correspond to a higher driving velocity of $3.5 \times 10^{-3} \mathrm{rad} / \mathrm{s}$. We observe that all the points fall on the same curve, which is not the case if the vertical displacement $\Delta h$ is plotted versus the horizontal slip distance $\Delta x=\beta M g / k \Delta \mu$ (see the inset in Fig. 10). This indicates that this compaction is not a 
pure geometrical effect due to the rolling of moving grains on a bed of fixed grains. In such a case, we expect a relation like $\Delta h=F(\Delta x)$, where $F$ represents an averaged grain profile. Likewise, it does not correspond to a loss of contact between the upper and lower part of the shear zone. In such a case, the relation would have been $\Delta h=\Delta x /\left(\beta \mu_{\text {slip }}\right)$, where $\mu_{\text {slip }}$ is the friction coefficient at slippage.

The description of a shear force dependent of the dilatancy and the sliding velocity $[13-15]$ is confirmed by the results shown on Fig. 10 since it implies that the coefficient of friction is a function of the dilatancy. One point remains to be investigated: does the dilatancy replace the contact time $\theta$ of the Dieterich-Ruina model [Eq. (5)] or is it a supplementary state variable that should be incorporated in the model [3]?

\section{Dilatancy during yielding}

In stick-slip experiments in solid friction [13], or for wet particles [14], it has been observed that a dilatancy occurs just before the slips, at the moment the creep becomes noticeable. This is not the case in our experiments, nor in those reported by Géminard et al. [16] with dry particles, for which the rate of dilatancy is maximum at the beginning of the stick phase. At low load (like $M=6.5 \mathrm{~kg}$, Fig. 3), the powder, before a large slip event, can slide over a distance much greater than a particle diameter whereas the dilatancy reaches a plateau. But when increasing the normal loading, this dilatancy has no time to come to equilibrium: the sample is still expanding at the moment it collapses. It is then not possible to relate the rate of dilatancy to the triggering of slip events.

\section{The slippage triggering}

The Dieterich-Ruina model, which well describes the transition to the small oscillatory behavior, does not explain this periodic collapse during the transition regime and what provokes it. After the instability threshold, the linear stability analysis suggests an exponential growth in time of the oscillatory behavior. The nonlinearities cause the amplitude of the oscillations to saturate. Here the oscillations during the unstable yielding grow until they destabilize the shear plane and make the granular medium reorganize. In the following, we investigate the onset of this new instability.

\section{Observation of a critical sliding velocity}

For all the experiments performed at $\omega_{0}$ $=3 \times 10^{-4} \mathrm{rad} / \mathrm{s}$, rupture always appears after a maximum of the friction force, as can be seen in Fig. 3, and in Fig. 11, left part, which zooms in on the shear behavior just before the slip. The slope at breakage $(d \mu / d t)_{\text {break }}$ is then negative. Looking more carefully, it can be observed that this slope decreases when increasing the loading mass and Fig. 12 shows the proportionality with $1 / M$. This result, together with Eq. (4), means that the angular velocity of the cover plate at breakage $\dot{\alpha}_{\text {break }}$ is the same for all these experiments. The sliding velocity at slippage $\omega^{*}$ is then constant and greater than $\omega_{0}$

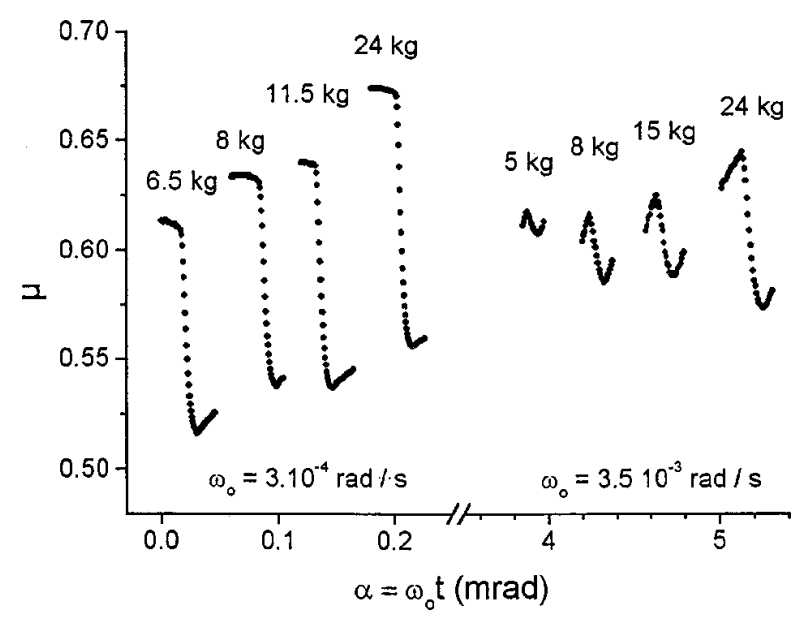

FIG. 11. Frictional coefficient $\mu$ as a function of angle of displacement $\omega_{0} t$ near big slip events for different normal loads and two velocities. Time interval between two points: $2 \mathrm{~ms}$.

$$
\begin{aligned}
\omega^{*} & =\omega_{0}-\dot{\alpha}_{\text {break }}=\omega_{0}-\frac{\beta}{\bar{R}} \frac{M g}{k}\left(\frac{d \mu}{d t}\right)_{\text {break }} \\
& =4.6 \pm 0.5 \times 10^{-4} \mathrm{rad} / \mathrm{s},
\end{aligned}
$$

where $\omega^{*}$ appears as the sliding velocity above which the powder destabilizes and compacts.

\section{High velocity shear experiment}

If $\omega^{*}$ is a material constant, then, when the shear driving velocity $\omega_{0}$ is higher than $\omega^{*}$, the rearrangements will occur before the slope of the curve $\mu(t)$ becomes negative (for $\left.\dot{\alpha}_{\text {break }}=\omega_{0}-\omega^{*}>0\right)$. This is indeed what is observed when performing experiments at $\omega_{0}=3.5 \times 10^{-3} \mathrm{rad} / \mathrm{s}$ : only small saw-tooth stick-slip oscillations (Figs. 13 and 11, right part) are observed. The medium cannot follow the velocity imposed.

We then observe, for this material, a critical sliding velocity (about $100 \pm 25 \mu \mathrm{m} / \mathrm{s}$ at the mean radius $\bar{R}$ ) above

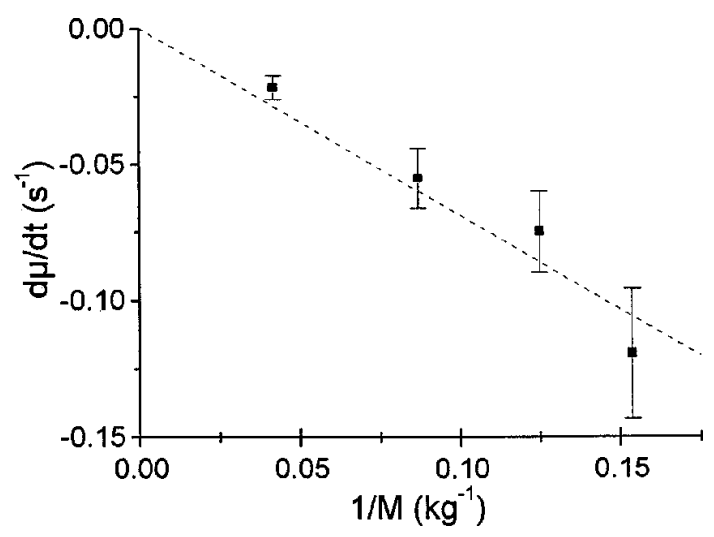

FIG. 12. Slope at breakage of the curve $\mu=f(t)$ of Fig. 11, left part as a function of the inverse of the normal load applied $1 / M$. (The error bars take into account both the dispersion of the measures and the discretization of the signal.) 


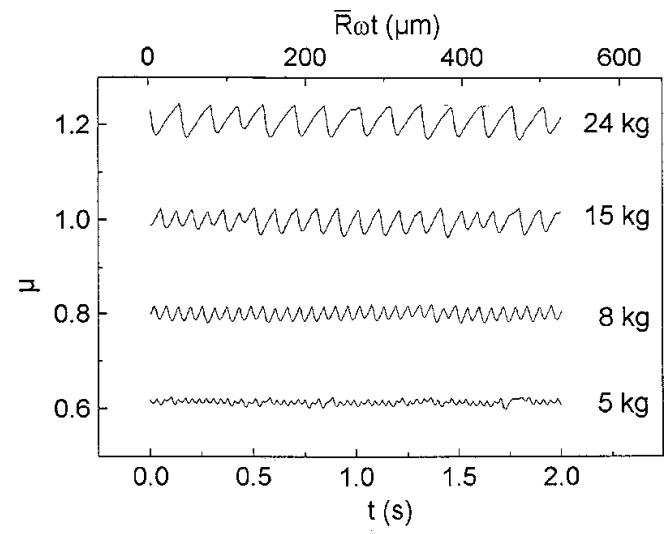

FIG. 13. Frictional coefficient $\mu$ versus time for different normal loads $M$, using the cell of inner radius $5 \mathrm{~cm}$ and outer radius of $10 \mathrm{~cm}$, and a driving velocity of $\omega_{0}=3.5 \times 10^{-3} \mathrm{rad} / \mathrm{s}$. (For clarity, the curves are shifted from each other by a jump of 0.2 .)

which the powder reacts by a slip rearrangement. The maximum sliding velocity appears to be a more relevant criterion than the creep or yield before slippage [10] or the dilatancy of the bed for explaining the occurrence of the big jumps in friction force. It remains to investigate the origin of this time dependency and its link to the velocity dependence of the friction coefficient.

\section{CONCLUDING REMARKS}

These preliminary observations on a sheared fine noncohesive silica gel show that the shear response of a powder presents strong analogies with solid friction. The observation of the transition from steady-state sliding to stick-slip instability for this powder gives a threshold in normal load or stiffness, which is well described by the Dieterich-Ruina model. Nevertheless, some special features, not seen in solid friction are also observed: when increasing the loading after the instability threshold, the shear is a succession of stick, yield, and slip phases. The ductile yielding is unstable and presents growing oscillations. It ends when the sliding velocity exceeds a critical value. The granular material then breaks as a fragile material. The origin of this velocity criterion remains unclear. It supposes the definition of a typical time under which the material has no time to accommodate the deformation. The way to improve the friction models, in order to take into account this behavior, remains to be investigated.

The dilatancy measurements have shown that the huge slips are associated with a reorganization of the powder bed that compacts by a fraction of a particle diameter. This compaction (Fig. 10) appears to be linked to the friction coefficient and not to the horizontal displacement. This point is in accordance with the models which attempt to link the friction coefficient with both the sliding velocity and dilatancy.

This paper still leaves open the question to finding relevant precursors that anticipate huge quake events whether in earth movements or in silo discharging. Close to the transition, the slippage may be preceded by an unstable yielding of arbitrary strain. Finally, it must be pointed out that our experimental setup, more representative of what occurs in storage silos, oversimplifies the behavior of a geological fault. In particular, the constant normal stress should be replaced by an elastic loading (normal stresses proportional to the dilatancy), which should be more realistic and lead to a greater complexity due to the formation of flying buttresses [17].

\section{ACKNOWLEDGMENTS}

It is a pleasure to thank Jean Rajchenbach, John A. Dodds, Guy Giraud, Eric Cervos, and Pierre Labourt-Ibarre for discussions and encouragement.
[1] A. W. Jenike, Bulletin $\mathrm{n}^{\circ} 123$, Engineering Experiment Station, The University of Utah, Salt Lake City, 1964.

[2] R. M. Nedderman, Statics and Kinematics of Granular Materials (Cambridge University, Cambridge, 1992).

[3] A. Ruina, J. Geophys. Res. 88, 10359 (1983).

[4] J. H. Dieterich, J. Geophys. Res. 84, 2161 (1979).

[5] C. Marone, Annu. Rev. Earth Planet Sci. 26, 643 (1998); Nature (London) 391, 69 (1998).

[6] C. Scholz, Nature (London) 391, 37 (1998).

[7] R. A. Bagnold, Proc. R. Soc. London, Ser. A 295, 219 (1966).

[8] S. B. Savage and M. Sayed, J. Fluid Mech. 142, 391 (1984).

[9] M. Hanes and D. L. Inman, J. Geophys. Res. 90, 3670 (1985).

[10] T. Baumberger, F. Heslot, and B. Perrin, Nature (London) 367, 544 (1994).
[11] T. Baumberger, F. Heslot, and B. Perrin, Phys. Rev. E 49, 4973 (1994).

[12] S. Nasuno, A. Kudrolli, and J. P. Gollub, Phys. Rev. Lett. 79, 949 (1997).

[13] D. M. Tolstö, Wear 10, 199 (1967).

[14] J. C. Géminard, W. Losert, and J. P. Gollub, Phys. Rev. E 59, 5881 (1999).

[15] F. Lacombe, S. Zapperi, and H. J. Herrmann, Eur. Phys. J. E 2, 181 (2000).

[16] W. Losert, J.-C. Géminard, S. Nasuno, and J. P. Gollub, Phys. Rev. E 61, 4060 (2000).

[17] E. Kolb, T. Mazozi, E. Clément, and J. Duran, Eur. Phys. J. B 8, 483 (1999). 